\title{
Linfoma intravascular tratado con anticuerpos monoclonales anti CD20: Descripción de un caso clínico
}

\author{
Jorge Alfaro $\mathrm{L}^{1,3}$, Arturo Espinoza $\mathrm{N}^{5}$, María Manríquez $\mathrm{A}^{5}$, \\ Leonor Moyano ${ }^{2}$, Néstor González $G^{3}$, Milton Larrondo $L^{1,3}$, \\ Gastón Figueroa $\mathrm{M}^{4}$. \\ Intravascular lymphoma treated with \\ anti CD20 monoclonal antibodies. \\ Report of one case
}

We report a 78 year old male with prostatism, that was subjected to a prostate biopsy. The pathological study showed a microvascular lymphocytic infiltration. Four months later, the patients presentd with reduced alertness, cough, dyspnea, fever and elevation of lactic dehydrogenase and erythrocyte sedimentation rate. Chest and abdominal CAT scans, bone marrow aspirate, protein electrophoresis and prostate specific antigen were normal. A re-evaluation of prostate biopsy showed an intravascular lymphoid infiltration, positive for CD45 and CD20, compatible with the diagnosis of intravascular lymphoma. Chemotherapy was started, but it was not tolerated by the patient and the response was partial. Therefore, treatment with monoclonal antibodies anti CD20 (Rituximab) was started. The tumor had a complete and prolonged (24 months) remission after the treatment (Rev Méd Chile 2004; 32: 1403-6).

(Key Words: Antibodies, monoclonal; CD20 antigens; Lymphoma, non-Hodgkin)

Recibido el 9 de marzo, 2004. Aceptado en versión corregida el 27 de agosto, 2004.

Laboratorio de Terapia Celular, Banco de Sangre ${ }^{1}$, Anatomía Patológica², Sección de Hematología ${ }^{3}$ y Sección de Medicina Interna ${ }^{4}$, Hospital Clínico de la Universidad de Chile. Servicio de Oncología y Anatomía Patológica de Clínica Dávila ${ }^{5}$. Santiago de Chile.

L os linfomas intravasculares constituyen una mara variedad de linfomas no Hodgkin (LNH). Se caracterizan por la proliferación intravascular de blastos linfoides, generalmente de estirpe $B$, sin que el compromiso ganglionar o extranodal

Correspondencia a: Dr. Jorge Alfaro L Laboratorio de Terapia Celular, Sección de Hematología, Hospital Clínico Universidad de Chile. Santos Dumont 999, Independencia, Santiago. Fono: 6788067. Fax: 6788071. E-mail: jalfaro@ns.hospital.chile.cl sea evidente. Esta variedad de linfoma puede afectar diversos órganos: piel, sistema nervioso central, próstata, corazón, bazo, riñón, glándulas adrenales, hígado, tracto genital y pulmón ${ }^{1}$. El diagnóstico es complejo debido a lo inespecífico de los síntomas, y una parte de los casos clínicos reportados están basados en estudios post mortem ${ }^{1,2}$. Presentamos el caso clínico de un paciente con LNH intravascular, con respuesta parcial a tratamientos con quimioterapia CHOP y VAD, 
quien entró en remisión completa después de un tratamiento con anticuerpos monoclonales anti $\mathrm{CD}^{20}$.

Caso clínico. Paciente de 78 años de edad, con antecedentes de diabetes mellitus tipo 2 en tratamiento con metformina, hipertensión arterial en tratamiento con diltiazem $30 \mathrm{mg}$ tres veces al día y cardiopatía coronaria tratada con ácido acetilsalicílico $100 \mathrm{mg} /$ día. Siete meses previo a su hospitalización se realizó una resección transuretral de próstata, cuya biopsia fue informada como adenoma prostático e infiltración linfocítica a nivel de microvasculatura. Estudio complementario: tomografía axial computada (TAC) de tórax, abdomen y pelvis, biopsia de médula ósea y mielograma fueron informados como normales, sin recibir el paciente tratamiento complementario. Cuatro meses previos al ingreso, el paciente refirió compromiso de su estado general y decaimiento. Estudio de antígeno prostático específico, ecocardiografía 2D, ecografía abdominal, electroforesis de proteínas plasmáticas, cadenas livianas, mielograma y radiografía de tórax, resultaron dentro de límites normales. Endoscopia digestiva alta: duodenitis con presencia de Helicobacter pylori.

Dos semanas previo a su ingreso refirió mialgias, fiebre, compromiso del estado general, baja de peso de $4 \mathrm{~kg}$, alteraciones del sensorio, con desorientación temporo-espacial, tos y expectoración mucosa. Recibió tratamiento con cefuroxime y azitromicina, sin observarse respuesta. Por agravamiento de sus condiciones generales se decidió su hospitalización.

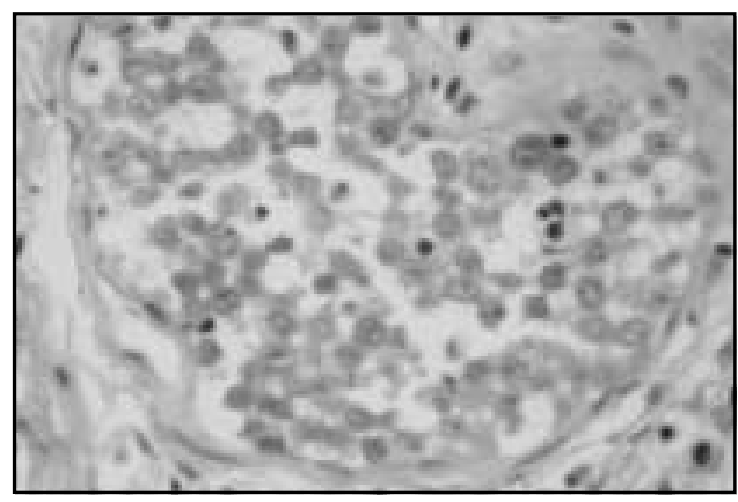

FiguRA 1. Hematoxilina-eosina (100x). Pared de vaso sanguíneo con abundantes blastos en el lumen.
Antecedentes: El paciente poseía el antecedente de apendicectomía y colecistectomía, y una fractura de cadera operada hacía 7 años. Examen físico: destacaba moderado compromiso de su estado general, presión arterial 130/80 mmHg, frecuencia cardíaca $88 / \mathrm{min}$, temperatura $36,7^{\circ} \mathrm{C}$ axilar. Desorientación, oscilaciones en su estado de conciencia, sin signos de focalización. Sin adenopatías periféricas. Mucosas sanas.

Laboratorio: Hematocrito 34,9\%, leucocitos 9.600/ $\mu \mathrm{L}$, neutrófilos $5.088 / \mu \mathrm{L}$, linfocitos $2.496 / \mu \mathrm{L}$, monocitos 2.016/ $\mu \mathrm{L}$, plaquetas $215.000 / \mu \mathrm{L}$, VHS 107 $\mathrm{mm} / \mathrm{h}$, proteína C reactiva 10,95 (valor normal $<1$ $\mathrm{U} / \mathrm{dL}$ ), láctico deshidrogenasa (LDH) $861 \mathrm{U} / \mathrm{L}$ (valor normal $<618 \mathrm{U} / \mathrm{L}$ ), función renal normal, función hepática normal. Antígeno prostático específico 1,89 (valor normal $<4 \mathrm{ng} / \mathrm{ml}$ ), ecocardiografía transesofágica normal, TAC tórax, abdomen y pelvis normal. Mielograma: Dentro de límites normales, a excepción de un pequeño grupo de blastos en una de las láminas.

Se revisó la biopsia de próstata tomada 8 meses antes y se observó un infiltrado intravascular linfoide, CD45 (+), CD20 (+) (Figuras 1 y 2), estableciéndose el diagnóstico de linfoma intravascular de células grandes. El paciente inició quimioterapia CHOP (ciclofosfamida, doxorrubicina, vincristina y prednisona) ${ }^{3}$ con buena respuesta clínica inicial; evolucionó asintomático, desapareciendo la fiebre y mejorando sus condiciones generales y mentales. Después del quinto ciclo de quimioterapia, el paciente volvió a presentar fiebre, compromiso del estado general, alteración del

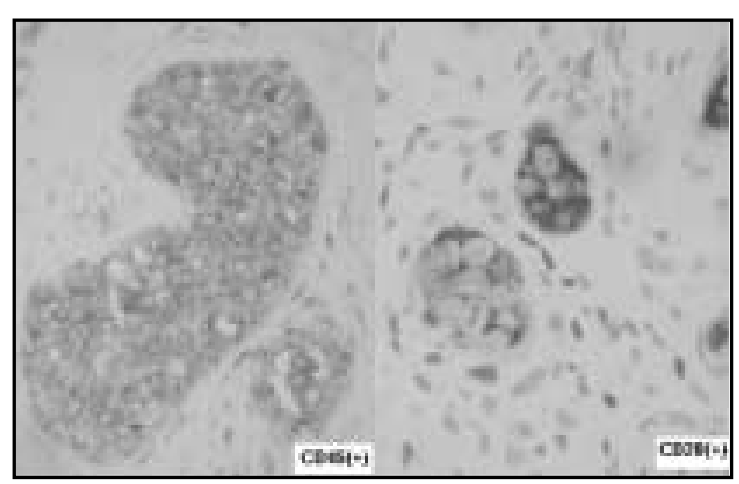

FIguRA 2. Izquierda: Tinción CD45 positiva (100x). Derecha: Tinción CD20 positiva (400x). 
sensorio y elevación de LDH y VHS. Nueva electroforesis de proteínas plasmáticas evidenció un pequeño peak monoclonal. Se cambió esquema de quimioterapia a VAD (vincristina, doxorrubicina, dexametasona) ${ }^{4}$ con lo cual mantuvo cierta estabilidad, pero con frecuentes descompensaciones previo al inicio de cada quimioterapia y mala tolerancia a la dexametasona por descompensación de sus glicemias. Después del tercer ciclo de quimioterapia $\mathrm{VAD}$, y estando el paciente en malas condiciones generales, con signos evidentes de actividad tumoral: fiebre, aumento de VHS, aumento de LDH y alteración de su estado de conciencia, se administró rituximab (anticuerpos monoclonales anti CD20. Mabthera Roche) $375 \mathrm{mg} / \mathrm{m}^{2}$ semanal por cuatro dosis. El paciente entró en remisión clínica de sus síntomas, normalización de $\mathrm{LDH}$, normalización de su estado mental y desaparición de la tos, manteniéndose en remisión clínica completa con 24 meses de seguimiento.

\section{DisCUSIÓN}

El linfoma intravascular fue descrito por primera vez en 1959 por L Pfleger, como una neoplasia endotelial denominada sangioendoteliomatosis proliferans systemisata», ha recibido además otras denominaciones como malignant angioendotheliomatosis»y «neoplastic endotheliosis». Actualmente el nombre más aceptado es el de linfoma intravascular $(\mathrm{LIV})^{5}$.

$\mathrm{Su}$ origen linfoide fue determinado en 1986 mediante estudio inmunohistoquímico ${ }^{5,6}$, siendo la mayoría de los casos descritos de origen $\mathrm{B}$ y, en menor porcentaje $\mathrm{T}^{7-9}$. Las manifestaciones clínicas observadas con mayor frecuencia son las alteraciones neurológicas (defectos focales, encefalopatía, neuropatía periférica) $)^{1,2,10-12}$. También son comu-

\section{REFERENCIAS}

1. Demirer T, Dail DH, Aboulafia DM. Four varied cases of intravascular lymphomatosis and a literature review. Cancer 1994; 73: 1738-45.

2. Yegappan S, Coupland R, Arber DA, Wang N, MiocinoVIC R, TubBs RR et aL. Angiotropic lymphoma: An nes las manifestaciones dermatológicas y fiebre. El diagnóstico diferencial de esta entidad debe ser con otras neoplasias intravasculares: carcinoma, melanoma maligno, síndrome de Sweet y neoplasia endotelial propiamente tal, siendo indispensable el estudio inmunohistoquímico.

Histológicamente, el LIV se caracteriza por una proliferación de células mononucleares en el lumen capilar, venular, arteriolar y de pequeñas arterias, con escaso compromiso del parénquima adyacente $^{2}$. Puede afectar cualquier órgano, aunque el compromiso de la médula ósea es infrecuente $^{13}$.

En el paciente descrito, existía un infiltrado prostático, como hallazgo incidental de una cirugía por adenoma prostático, cuya correcta interpretación clínica fue dada con posterioridad, debido a la ausencia de compromiso ganglionar 0 de otro parénquima en los estudios de extensión. Presentó posteriormente fiebre, alteraciones del sensorio, tos e hipoxemia, hallazgos frecuentes en esta entidad ${ }^{14-16}$.

En el estudio de laboratorio se pudo observar una elevación de la enzima deshidrogenasa láctica (LDH) y la velocidad de eritrosedimentación (VHS).

Cuando el diagnóstico es hecho precozmente, el tratamiento con poliquimioterapia logra un cierto porcentaje de respuestas completas persistentes ${ }^{17}$. El paciente referido recae durante la terapia con CHOP y no logra buen control de la enfermedad con VAD. El uso de anticuerpos monoclonales anti CD20 fue utilizado como rescate, obteniéndose una remisión completa y prolongada (24 meses).

El tratamiento con anticuerpos monoclonales anti CD20 ha sido probado con éxito en el tratamiento de LNH de estirpe B y se ha posicionado como un importante avance terapéutico, destacando por su eficacia y baja toxicidad, como se ejemplifica en este caso ${ }^{18,19}$.

immunophenotipically and clinically heterogeneous lymphoma. Mod Pathol 2001; 14: 1147-56.

3. Fisher R, Gaynor E, Dahlberg S, O ken M, Grogan T, MIzE E ET AL. Comparison of a standard regimen (CHOP) with three intensive chemotherapy regimens for advanced non-Hodgkin's lymphoma. N Engl J Med 1993; 328: 1002-6. 
4. Barlogie B, Smith L, Aiexanian R. Effective treatment of advanced multiple myeloma refractory to alkylating agents. N Engl J Med 1984; 310: 1353-6.

5. Wick M, Mius S, Scheithauer B, Cooper P, Davitz M, PARKInson K. Reassessment of malignant angioendotheliomatosis. Evidence in favor of its reclassification as «ntravascular lymphomatosis». Am J Surg Pathol 1986; 10: 112-23.

6. Sheibani K, Battifora H, Winberg C, Burke J, BenEZRA J, Eunger G ET AL. Further evidence that «Malignant angioendotheliomatosis» is an angiotropic large-cell lymphoma. N Engl J Med 1986; 314: 943-8.

7. Lakhani SR, Hulman G, Hall JM, Slack DN, Sloane JP. Intravascular malignant lymphomatosis (angiotropic large-cell lymphoma). A case report with evidence for T-cell lineage with polymerase chain reaction analysis. Histopathology 1994; 25: 283-6.

8. Au WY, Shek WH, Nichoшs J, TSE KM, Todd D, Kwong YL. T-cell intravascular lymphomatosis (angiotropic large cell lymphoma): association with Epstein-Barr viral infection. Histopathology 1997; 31: 563-7.

9. MaLicki DM, Suh YK, Fuller GN, Shin SS. Angiotropic (intravascular) large cell lymphoma of T-cell phenotype presenting as acute appendicitis in a patient with acquired immunodeficiency syndrome. Arch Pathol Lab Med 1999; 123: 335-7.

10. SienkNeCht C, Whetsell W, Poшock P. Intravascular malignant lymphoma ( Malignant angioendotheliomatosis») mimicking primary angiitis of the central nervous system. J Rheumatol 1995; 22: 1769-70.

11. Cheng F, Tsui W, Yeung WC, Ip L, Ng C. Intravascular lymphomatosis: a case presenting with encephalomyelitis and reactive haemophagocytic syndrome diagnosed by renal biopsy. Histopathology 1997; 31: 552-4.

12. LEVIN K, LuTZ G. Angiotropic large-cell lymphoma with peripheral nerve and skeletal muscle involvement: Early diagnosis and treatment. Neurology 1996; 47: 1009-11.

13. Іто M, Kim $\mathrm{Y}$, Choi JM, Ozawa H, Fujino $M$. Prevalence of intravascular large B-cell lymphoma with bone marrow involvement at initial presentation. Int J Hematol 2003; 77: 159-63.

14. Deusch E, Mayr A, Hobisch-Hagen P, Fend F, Mutz N, Bangerl I et al. Angiotropic lange B-cell lymphoma misdiagnosed as urosepsis with multiple organ dysfunction syndrome. Acta Anaesthesiol Scand 1999; 43: 100-3.

15. IP M, CHAN KW, CHAN IK. Systemic inflammatory response syndrome in intravascular lymphomatosis. Intensive Care Med 1997; 23: 783-6.

16. Wald JG, Hong YG, Cox JE, McCabe KM, O’Brien KE, Auerton JP et al. Pulmonary intravascular lymphomatosis. Presentation with dyspnea and air trapping. Chest 1999; 115: 1207-10.

17. DiGiuseppe JA, Neison WG, SeifTer EJ, BoItnott JK, ManN RB. Intravascular lymphomatosis: A clinicopathologic study of 10 cases and assessment of response to chemotherapy. J Clin Oncol 1994; 12: 2573-9.

18. Czuczman M, GriLo-Lopez A, White C, Salah M, Gordon L, LoBuglio AF ET AL. Treatment of patient with low grade B-cell lymphoma with the combination of chimeric anti-CD20 monoclonal antibody and CHOP chemotherapy. J Clin Oncol. 1999; 17: 268-76.

19. Coiffier B, Lepage E, Brière J, Herbrecht R, Tily H, BouabDalah R ET aL. CHOP chemotherapy plus rituximab compared with $\mathrm{CHOP}$ alone in elderly patients with diffuse large B-cell lymphoma. N Engl J Med 2002; 346: 235-42. 